\title{
Análisis de los estilos y estrategias de aprendizaje en estudiantes de psicología
}

Ulises Delgado Sánchez ${ }^{1}$, Fernanda Gabriela Martínez Flores ${ }^{1}$ y Angélica Flores López ${ }^{1}$

\section{Resumen}

Los estudiantes universitarios desarrollan estilos y estrategias de aprendizaje idiosincrásicas. La evidencia con estudiantes de Licenciatura en Psicología es limitada. El objetivo del presente trabajo fue identificar estilos y estrategias de aprendizaje en estudiantes de psicología de la UNAM-FEs Iztacala y la UAEM-Campus Chamilpa, determinando el grado en que ambos predicen el logro escolar de los mismos. Se aplicó el ACRA, que evalúa: estrategias cognitivas y de control, estrategias de apoyo y hábitos de estudio; y el CHAEA, que identifica los estilos pragmático, teórico, reflexivo y activo, a 300 estudiantes de la UAEM y 336 de la UNAM. Los resultados muestran coincidencias en estilos y estrategias, con diferencias significativas en el estilo teórico y las estrategias de apoyo, respectivamente. Se identificó una correlación positiva débil entre el promedio y el estilo activo (rho $=0.08, p=0.02)$ y reflexivo ( $r h o=0.10, p=0.01$ ), así como una correlación positiva débil con la estrategia de apoyo ( $r h o=0.08, p=0.04)$.

1 Universidad Autónoma del Estado de Morelos. 
Palabras clave: estilos de aprendizaje, estrategias de aprendizaje, logro escolar, estudiantes universitarios.

\section{Introducción}

En los últimos años se ha incrementado el interés de los investigadores en torno a las estrategias y los estilos de aprendizaje que desarrollan los estudiantes (Valle, González, Cuevas \& Fernández, 1998). Los estilos de aprendizaje han sido definidos por James. W. Keffe como comportamientos cognitivos, afectivos y psicológicos que sirven como indicadores relativamente estables de cómo los alumnos perciben, interactúan y responden al ambiente de aprendizaje (Felder \& Spurlin, 2005). Por otra parte, las estrategias de aprendizaje se consideran como habilidades que los estudiantes adoptan al estudiar (Cassidy, 2004).

Los estilos de aprendizaje han sido categorizados y operacionalizados desde diferentes modelos, siendo el más influyente el propuesto por Kolb (1981), del cual derivan tanto el Learning Style Inventory (LSI), que clasifica los estilos de los estudiantes en: convergente, divergente, acomodador y asimilador, como el Cuestionario Honey-Alonso (CHAEA), que divide los estilos en cuatro: activo, reflexivo, pragmático y teórico; cuya aplicación cubre principalmente España y países de habla hispana y portuguesa (Cassidy, 2004; Castro \& Castro, 2005; López \& Ballesteros, 2003; Ruiz, Gamboa \& Arrieta, 2006).

La evidencia generada a través de la aplicación del CHAEA ha permitido una comprensión amplia de los estilos de aprendizaje y su relación con diferentes variables en estudiantes de bachillerato. Sin embargo, la información aún es escasa en el nivel universitario. López y Ballesteros (2003) encontraron en la carrera de enfermería que el estilo predominante es el reflexivo, seguido del teórico y del activo. Por otra parte, Esguerra y Guerrero (2010) encontraron en estudiantes de psicología un predominio del estilo reflexivo y una menor presencia del activo y pragmático, aunque con variaciones a través de los semestres de la carrera. En un estudio comparativo entre diferentes carreras, las cuales son psicología, contaduría, derecho, ingeniería 
en computación, informática administrativa y administración, se identificó que existía una tendencia moderada hacia los estilos activo, teórico y pragmático, siendo el reflexivo el más bajo, a excepción de la carrera de psicología que fue moderado (Juárez, Rodríguez \& Luna, 2012).

En cuanto a las estrategias de aprendizaje, también impera una variedad de modelos, tales como el de J. B. Biggs, llamado 3P, el cual identifica estrategias de tipo superficial, profundo y de logro, por medio de los instrumentos Learning Process Questionnaire (LPQ) y el Study Process Questionnaire (SPQ) (Biggs, 1991; Biggs, Kember \& Leung, 2001; López \& Falchetti, 2009). Entre los países de habla hispana, destaca el instrumento ACRA, que corresponde a las estrategias de adquisición, codificación, recuperación y de apoyo de información (Beltrán, 2003; Correa, Castro \& Lira, 2004). Con este último se ha identificado que estudiantes de ingeniería agronómica usan mayormente estrategias de codificación, seguida de las de apoyo, adquisición y recuperación (Albo, 2012). También en estudiantes de la Licenciatura en Educación Preescolar, se encontró una asociación estadísticamente significativa con el uso de estrategias de adquisición y el promedio acumulado, seguida de las estrategias de recuperación y apoyo que no presentaron diferencias estadísticamente significativas (Delgado, Cárdenas, Flores \& Guzmán, 2014). Por otra parte, con estudiantes de pedagogía en enseñanza media, se encontró que $65 \%$ de la muestra no usa o usa sólo algunas veces las estrategias de adquisición de información; que 50\% usa estrategias para la codificación de información; 45\% para la recuperación de información; y 45\% no maneja o sólo maneja algunas veces estrategias de apoyo al procesamiento de información (Correa, Castro \& Lira, 2004).

La información obtenida hasta el momento parece indicar que cada área del conocimiento, debido a sus diferentes enfoques, procedimientos y objetivos particulares, conduce a que en los estudiantes predomine un estilo característico. Varias investigaciones han respaldado que sí existe una cultura relativamente homogénea disciplinariamente, pero heterogénea a través de diferentes disciplinas (Acevedo, Chiang, Madrid, Reinicke \& Rocha, 2009; Esguerra \& Guerrero, 2010; Kolb, 1981; Laugero, 
Balcaza, Salinas \& Craveri, 2009; López \& Ballesteros, 2003; Ochoa \& Aragón, 2004; Ventura, Moscoloni \& Gagliardi, 2012). Esto parece apuntar por igual a las estrategias de aprendizaje de los alumnos, sin embargo, no parece haber evidencia suficiente, y en grado mucho menor, información de carácter comparativo de la relación entre ambas variables en estudiantes de alguna licenciatura en diferentes universidades.

El objetivo central del presente estudio es realizar un análisis comparativo de los estilos y estrategias de aprendizaje presentes en estudiantes de psicología de dos diferentes universidades de México. Los objetivos particulares son los siguientes: 1) identificar los estilos y las estrategias de aprendizaje con mayor presencia en estudiantes de psicología en la UNAM-FES Iztacala y la UAEM-Campus Chamilpa; 2) comparar los valores de los estilos y estrategias encontrados, a fin de establecer la presencia/ausencia de algún perfil común; y 3) determinar el grado en que ambos aspectos predicen el logro escolar expresado por el promedio acumulado de los estudiantes.

\section{Método}

Participantes

636 alumnos universitarios; 300 estudiantes de la Facultad de Psicología de la Universidad Autónoma del Estado de Morelos Campus Norte y 336 estudiantes de la carrera de psicología de la Universidad Nacional Autónoma de México FEs-Iztacala. Todos los alumnos participaron voluntariamente y su selección fue intencional, abarcando los diferentes semestres de cada carrera durante el periodo lectivo 2014-2.

Instrumentos

Se aplicó el cuestionario de Honey-Alonso de estilos de aprendizaje (CHAEA), el cual es un cuestionario dicotómico que está constituido por 80 ítems agrupados en cuatro dimensiones: activo, pragmático, reflexivo y teórico. Del cuestionario sobre estrategias de aprendizaje (ACRA), se utilizó su versión abreviada que valora tres dimensiones: estrategias cognitivas y de control del 
aprendizaje (D1A), estrategias de apoyo al aprendizaje (D2A) y hábitos de estudio (D3A); este instrumento es una escala tipo Likert, donde los puntajes máximos a obtener en la D1A son de 100 puntos, en la D2A de 56 puntos y en la D3A de 20 puntos.

\section{Procedimiento}

Tres colaboradores entrenados aplicaron los cuestionarios ACRA y CHAEA aprovechando los grupos escolares existentes en cada Facultad, a partir del consentimiento de los profesores responsables y de los alumnos. Complementariamente, a cada estudiante se le requirió indicar su promedio acumulado hasta el semestre cursado. En cada centro educativo se procuró muestrear una cantidad proporcional de alumnos por cada año de avance en la carrera. La integración de la información y el procesamiento estadístico se llevó a cabo con el programa SPSS v.22.

\section{Resultados}

Se obtuvieron las medias de cada estilo de aprendizaje de los estudiantes de cada Facultad. Los valores identificados en los estudiantes de la UAEM fueron de 8.8 en activo, 5.1 en reflexivo, 7.4 en teórico y 7.3 en pragmático. Los estudiantes de la UNAM presentaron medias de 9.0 en activo, 5.2 en reflexivo, 7.9 en teórico y 7.6 en pragmático (ver Figura 1). Se encontraron diferencias estadísticamente significativas en el estilo teórico, con una $t_{(627)}=-2.02, p=$ 0.04 , presentando mayores puntajes los estudiantes de la UNAM.

De manera análoga, fueron calculadas las medias para las estrategias de aprendizaje de los estudiantes da cada Facultad. Los estudiantes de la UAEM mostraron los siguientes puntajes: D1A: 73.12, D2A: 39.37, D3A: 15.75; los estudiantes de la UNAM presentaron los siguientes valores: D1A: 74.55, D2A: 41.50, D3A: 15.59. Se encontraron diferencias estadísticamente significativas en la D2A, con una $t_{(629)}=-3.71, p=0.00$, presentando mejores puntajes la UNAM. En general, la media de promedio para la UAEM fue de 7.93 y para la UNAM de 8.31, siendo esta diferencia estadísticamente significativa: $t_{(618)}=-2.93, p=0.00$. Existe una correlación positiva débil entre el promedio con el estilo activo 
(rho $=0.08, p=0.02)$ y reflexivo $(r h o=0.10, p=0.01)$, así como una correlación positiva débil con la estrategia D2A (rho $=0.08$, $p=0.04)$.

\section{Estilos de aprendizaje en estudiantes de psicologia}

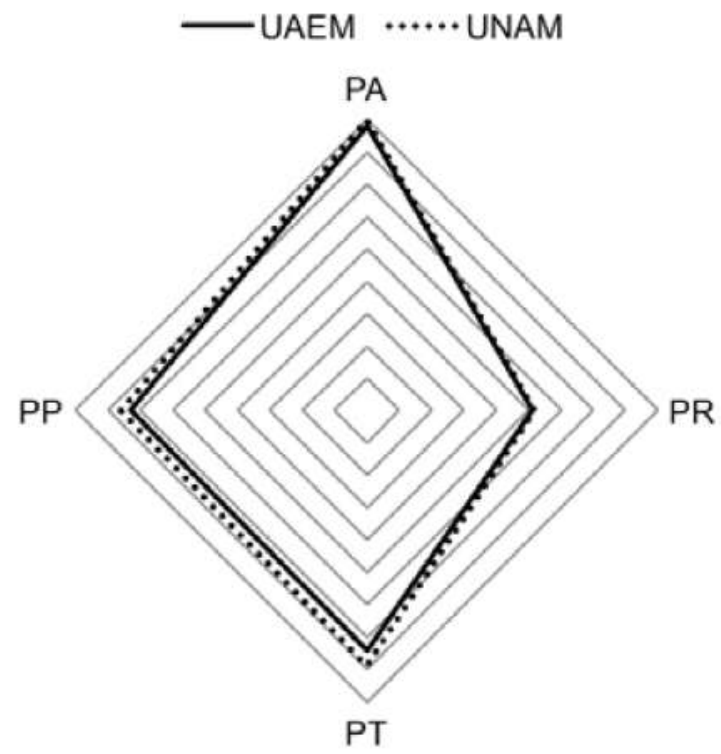

Figura 1. Dispersión de los estilos de aprendizaje con los puntajes obtenidos en CHAEA

\section{Discusión}

El objetivo del presente estudio fue realizar un análisis comparativo de los estilos y estrategias de aprendizaje presentes en estudiantes de psicología de dos diferentes universidades en México. Se encontró que los estudiantes de psicología, tanto de la UAEM como de la UNAM, presentan el mismo perfil de los estilos de aprendizaje, estando de manera jerárquica de uso el estilo activo, seguido del pragmático, teórico y reflexivo. Estos resultados contrastan con los reportados por Esquerra y Guerrero (2010), pero son coincidentes con los estudios de Juárez, Rodríguez y Luna (2012), en tanto que ser activo y pragmático parecen caracterizar al estudiante de psicología exitoso. En cuanto a las estrategias de aprendizaje, presentaron mayores puntajes los alumnos tanto de la UAEM como de la UNAM, en comparación con los estudios de Correa, Castro y Lira (2004). Las estrategias de apoyo correlacionaron 
con el promedio, coincidiendo con el estudio realizado por Delgado, Cárdenas, Flores y Guzmán (2014), quienes evaluaron dicha relación en grupos de la Licenciatura en Educación Básica.

Se encontró homogeneidad en la presencia de los estilos y las estrategias de aprendizaje de los estudiantes de psicología de la UAEM y la UNAM, lo que pondría de manifiesto que la estructura curricular, aún y cuando presenta diferencias, no genera una divergencia notable en los estilos y estrategias. En estudios posteriores, se podrían identificar, si existen, diferencias significativas de los estilos y estrategias de aprendizaje entre los estudiantes que se encuentran cursando semestres diferentes, así como diferencias en cuanto al sexo.

\section{Referencias}

Acevedo, P., Chiang, S., Madrid, V., Reinicke, S. \& Rocha, O. (2009). Estrategias de aprendizaje en alumnos universitarios y de enseñanza media. Revista de Estilos de Aprendizaje, 4(4), 138-155.

Albo, G. (2012). ACRA: escalas de estrategias de aprendizaje de los estudiantes del curso de producción animal I. Facultad de Ciencias Agrarias y Forestales (UNLP). IV Congreso Nacional y III Congreso Internacional de Enseñanza de las Ciencias Agropecuarias, Argentina.

Beltrán, J. (2003). Estrategias de aprendizaje. Revista de Educación. 332, 55-73.

Biggs, J. (1991). Approaches to Learning in Secondary and Tertiary Students in Hong Kong: Some Comparative Studies. Educational Research Journal, 6, 27-39.

Biggs, J. B., Kember, D. \& Leung, D. Y. (2001). The Revised Two Factor Study Process Questionnaire: R-SPQ-2F. British Journal of Educational Psychology. 71, 133-149.

Cassidy, S. (2004). Learning Styles: An overview of theories, models, and measures. Educational Psychology: An International Journal of Experimental Educational Psychology, 24(4), 419-444. Doi: 10.1080/0144341042000228834. 
Castro, S. \& Castro, G. (2005). Los estilos de aprendizaje: una propuesta para su implementación. Revista de Investigación. 58, 83-102.

Correa, M., Castro, E. \& Lira, H. (2004). Estudio descriptivo de las estrategias cognitivas y metacognitivas de los alumnos y alumnas de primer año de pedagogía en enseñanza media de la universidad Bío-Bío. Theoria, 13, 103-110.

Delgado, U., Cárdenas, K., Flores, C. \& Guzmán, M. (2014). Disposiciones para el aprendizaje y logro académico en estudiantes universitarios. Congreso Iberoamericano de Ciencia, Tecnología, Innovación y Educación. Buenos Aires, Argentina, 12, 13 y 14 de noviembre.

Esguerra, G. \& Guerrero, P. (2010). Estilos de aprendizaje y rendimiento académico en estudiantes de psicología. Diversas Perspectivas en Psicología, 6(6), 97-109.

Felder, R. \& Spurlin, J. (2005). Applications, Reliability and Validity of the Index of Learning Styles. International Journal of Engineering Education, 21(1), 103-112.

Juárez, C., Rodríguez, G. \& Luna, E. (2012). El cuestionario de estilos de aprendizaje CHAEA y la escala de estrategias de aprendizaje ACRA como herramienta potencial para la tutoría académica. Revista Estilos de Aprendizaje, 10(10), 1-31.

Kolb, D. A. (1981). Learning Styles and Disciplinary Differences. United States of America: The Modern American Collage.

Laugero, L., Balcaza, G., Salinas, N. \& Craveri, A. (2009). Una indagación en el estilo de aprendizaje de los alumnos en distintos momentos de su vida universitaria. Revista de Estilos de Aprendizaje, 4(4), 122-137.

López, C. \& Ballesteros, C. (2003). Evaluación de los estilos de aprendizaje en estudiantes de enfermería mediante el cuestionario CHAEA. Enfermería Global, 3, 1-12.

López, M. \& Falchetti, E. (2009). Estilos de aprendizaje. Relación con motivación y estrategias. Revista de Estilos de Aprendizaje, 4(4).

Ochoa, S. \& Aragón, L. (2004). La alfabetización en la universidad y su relación con las estrategias de aprendizaje. Pensamiento Psicológico, 2, 9-36 
Ruiz, B., Trillos Gamboa, J., Morales Arrieta, J. (2006). Estilos de aprendizaje y rendimiento académico en estudiantes universitarios. Revista Galego-Portuguesa de Psicoloxía e Educación, 13(11-12), 441-457.

Valle, A., González, C., Cuevas, G. \& Fernández, S. (1998). Las estrategias de aprendizaje: características básicas y su relevancia en el contexto escolar. Revista de Psicodidáctica, 6, 53-68. Recuperado de: http://www.redalyc.org/articulo. oa?id=17514484006.

Ventura, C., Moscoloni, N. \& Gagliardi, R. (2012). Estudio comparativo sobre los estilos de aprendizaje de estudiantes universitarios argentinos de diferentes disciplinas. Psicología desde el Caribe, 29(2), 276-304. 